\title{
Diet and Metformin in PCOS
}

\author{
Nisha Thakre ${ }^{1}$ and Anil Gudi ${ }^{2 *}$ \\ ${ }^{1}$ Clinical Research Fellow, Homerton University Hospital, London, UK \\ ${ }^{2}$ Director, Homerton University Hospital, London, UK \\ *Corresponding Author: Anil Gudi, Director, Homerton University Hospital, London, UK.
}

Received: July 01, 2019; Published: July 24, 2019

DOI: $10.31080 /$ ASNH.2019.03.0380

\section{Diet and PCOS}

PCOS creates an insulin resistant state and can have significant metabolic effects. There is a huge amount of information of decreasing weight and its impact of reducing the symptoms and the biochemical aspects of PCOS. What is less know is whether dietary interventions are effective for improving weight loss, metabolic, fertility and emotional well being. Whether specific dietary composition in lifestyle intervention is successful is controversial though it is marketed extensively.

Having reviewed the recent evidence which reviewed, outcomes from a high protein diet to a high carbohydrate diet found no evidence in for the majority of anthropometric, metabolic, fertility and emotional well being. The type of diet was not found to be effective in these studies. It was found that regardless of the type of diet, diet aimed at reducing weight was of benefit to women with PCOS. 2 large systematic reviews showed that there was no benefit with a specific diet and hormone level changes as well as insulin changes did not predict response. It was noted that weight loss was possible and patient complaint with low fat diet and reduced energy diets, though adding different micronutrient content seemed unjustified $[2,3]$.

Is metformin alone, or in combination, effective for management of PCOS?

Metformin is a low cost, readily available medication that has been extensively used as an insulin sensitiser for over seven decades in DM2 and for several decades in PCOS. Insulin resistance is documented on clamp studies in $75 \%$ of lean women and $95 \%$ of overweight women and addressing this has underpinned the use of metformin in PCOS. Metformin is currently widely used by women with PCOS, yet the efficacy of metformin in terms of improving clinical outcomes remains uncertain. Side effects do cause concern, and metformin use in PCOS is generally off label.

In PCOS, evidence indicates that metformin is effective overall in improving weight but I suspect that this is mainly due to the side effects of metformin; nausea, vomiting and diarrhoea!

Can metformin work with irregular cycles?

There is inadequate evidence to make a recommendation about the use of metformin for irregular menstrual cycles. Any efficacy in regularising menstruation usually takes at least 5 months. Weight loss has proved more efficient than metformin in restoring menstrual regularity.

The latest PCOS guidelines make the following recommendations regarding the use of metformin for non-conceptional indications:

- Metformin in addition to lifestyle, should be considered in adult women with PCOS with BMI $\geq 25 \mathrm{~kg} / \mathrm{m} 2$ for management of weight and metabolic outcomes.

- Metformin in additional to lifestyle, could be considered in adolescents with a clear diagnosis of PCOS or with symptoms of PCOS before the diagnosis is made.

- Metformin may offer greater benefit in high metabolic risk groups including those with diabetes risk factors, impaired glucose tolerance or high-risk ethnic groups.

The following should be considered when metformin is prescribed:

- Adverse effects, including gastrointestinal side-effects that are generally dose dependent and self-limiting, need to be the subject of individualised discussion

- $\quad$ Starting at a low dose, with 500mg increments 1-2 weekly and extended release preparations may minimise side effects 
- Metformin use appears safe long-term, based on use in other populations, however ongoing requirement needs to be considered and use may be associated with low vitamin B12 levels

- Use is generally off label and health professionals need to inform women and discuss the evidence, possible concerns and side effects.

Although metformin has been successful for the treatment of hirsutism, its main indication in PCOS has been for the restoration of ovulation. Metformin has a modest beneficial effect, at least in normal weight women, in increasing the frequency of ovulation. It is less effective than either clomiphene citrate (CC) or letrozole when used as monotherapy for induction of ovulation, pregnancy and live birth. The addition of metformin to CC in therapy naïve patients is no better than CC alone although when added to CC in CC resistant patients, metformin is capable of inducing ovulation and pregnancy in some cases and this has been suggested as a strategy before moving on to gonadotrophin therapy. Metformin is less effective in restoring ovulation in overweight women. The suggested capability of metformin in PCOS to improve miscarriage rates has not materialised.

\section{Metformin and teratogenicity}

Metformin has no proven teratogenicity and has been given during pregnancy with no apparent ill effects. It may decrease the incidence of gestational diabetes, macrosomia and pre-eclampsia.

\section{Metformin in adolescence}

Its use for adolescent PCOS is effective but controversial as is its use to avoid the long term sequelae of PCOS such as diabetes and cardio-vascular disease.

\section{Bibliography}

1. International evidence-based guideline for the assessment and management of polycystic ovary syndrome (2018).

2. Johnston BC., et al. "Comparison of weight loss among named diet programs in overweight and obese adults: a meta-analysis". JAMA 312.9 (2014): 923-933.

3. Gardner CD., et al. "Effect of low-fat vs low-carbohydrate diet on 12-month weight loss in overweight adults and the association with genotype pattern or insulin secretion: The dietfits randomized clinical trial". JAMA 319 (2018): 667-679.

\section{Volume 3 Issue 8 August 2019}

\section{(c) All rights are reserved by Nisha Thakre and Anil}

\section{Gudi.}

